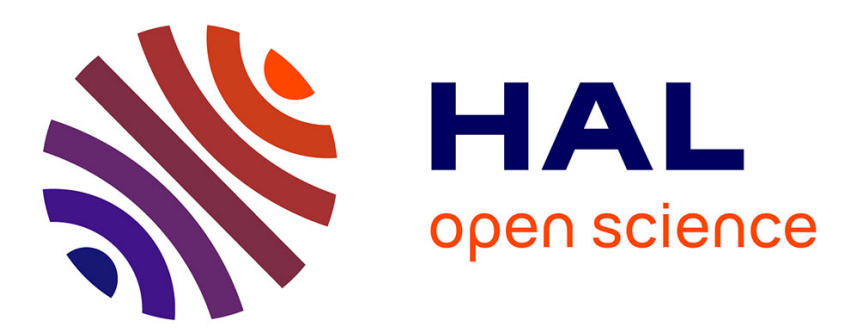

\title{
Amélioration de la stabilité d'un générateur électrostatique haute tension
}

\author{
G. Balossier, E. Mérienne, D. Genotel
}

\section{To cite this version:}

G. Balossier, E. Mérienne, D. Genotel. Amélioration de la stabilité d'un générateur électrostatique haute tension. Revue de Physique Appliquée, 1978, 13 (6), pp.313-316. 10.1051/rphysap:01978001306031300 . jpa-00244456

\section{HAL Id: jpa-00244456 https://hal.science/jpa-00244456}

Submitted on 1 Jan 1978

HAL is a multi-disciplinary open access archive for the deposit and dissemination of scientific research documents, whether they are published or not. The documents may come from teaching and research institutions in France or abroad, or from public or private research centers.
L'archive ouverte pluridisciplinaire HAL, est destinée au dépôt et à la diffusion de documents scientifiques de niveau recherche, publiés ou non, émanant des établissements d'enseignement et de recherche français ou étrangers, des laboratoires publics ou privés. 


\title{
AMÉLIORATION DE LA STABILITÉ D'UN GÉNÉRATEUR ÉLECTROSTATIQUE HAUTE TENSION
}

\author{
G. BALOSSIER, E. MÉRIENNE et D. GENOTEL \\ Laboratoire de Microscopie Electronique, Faculté des Sciences, \\ B. P. 34751062 Reims Cedex France
}

(Reçu le 15 décembre 1977, révisé le 6 mars 1978, accepté le 13 mars 1978)

\begin{abstract}
Résumé. - Nous décrivons un dispositif peu onéreux qui permet d'améliorer la stabilité d'un générateur électrostatique haute tension. L'instabilité relative en tension $\Delta V / V$ se trouve ainsi ramenée de $2 \times 10^{-5}$ à $2 \times 10^{-6}$ pour des fréquences allant de 0 à plus de $1000 \mathrm{~Hz}$.
\end{abstract}

\begin{abstract}
We describe an inexpensive electronic device which allows to reduce the instability of a $400 \mathrm{kV}$ electrostatic voltage generator. The relative instability $\Delta V / V$ is reduced from $2 \times 10^{-5}$ to $2 \times 10^{-6}$ in a range of frequencies from 0 to more than $1000 \mathrm{~Hz}$.
\end{abstract}

1. Introduction. - Dans un microscope électronique à transmission, le générateur haute tension doit être stable pour faire des observations en haute résolution $[1,2,3,4,5]$ : par exemple, pour une tension d'accélération des électrons $V=200 \mathrm{kV}$ et avec un coefficient d'aberration chromatique de la lentille objectif $C_{\mathrm{c}}=1,5 \mathrm{~mm}$, l'instabilité relative $\Delta V / V$ de la haute tension doit être meilleure que $2 \times 10^{-6}$ si on désire mettre en évidence avec un contraste de $10 \%$ un pouvoir séparateur de l'ordre de $2 \AA$.

Le microscope électronique supraconducteur $400 \mathrm{kV}$ que nous avons construit [6] est alimenté par un générateur électrostatique SAMES du type SAMTRON KS 400. L'instabilité de ce générateur est environ $\Delta V / V=2 \times 10^{-5}$. Nous avons donc entrepris de réduire cette instabilité afin qu'elle ne limite pas le pouvoir de résolution de notre microscope.

Nous avions déjà réalisé un premier dispositif de contre-réaction à lecture capacitive [7]. Le facteur de correction de ce dispositif étant trop faible aux basses fréquences, nous lui avons adjoint en série un système de contre-réaction à lecture résistive. Nous décrivons ci-après ce second dispositif et nous présentons les améliorations obtenues.

2. Principe de la stabilisation. - Nous présentons le montage réalisé sur la figure 1 . Il consiste en une association en série de deux contre-réactions, l'une à lecture capacitive placée entre la masse et une masse flottante $M_{1}$, l'autre à lecture résistive placée entre $M_{1}$ et la masse flottante $M$ du générateur HT.

Pour éviter tout risque d'interaction entre les deux contre-réactions nous avons séparé les bandes passantes des deux systèmes. Un filtre $\mathrm{RC}$ placé au niveau de l'étage amplificateur de la contre-réaction résistive

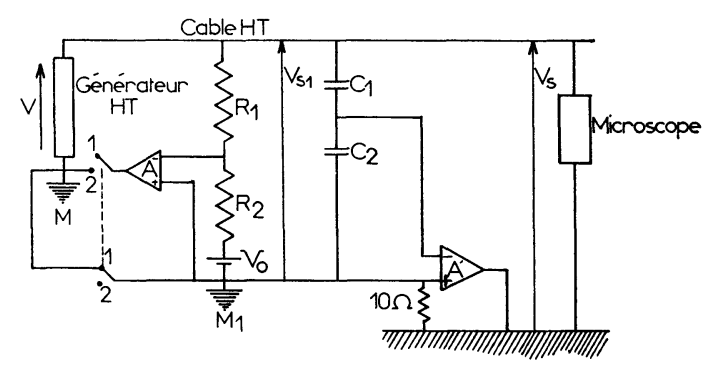

Fig. 1. - Solution adoptée comportant deux dispositifs de contre-réaction l'un à lecture résistive, l'autre à lecture capacitive, les deux étant montés en série.

[Selected solution with two series-feedback circuits; the one with a resitif coupling; the other with a capacitive coupling. Both are connected in series.]

limite son domaine d'action aux fréquences inférieures à quelques Hertz (Fig. 2). Dans cette gamme de fréquences l'action de la contre-réaction capacitive est considérablement réduite à cause du couplage capacitif (Fig. 3, courbe II).

De plus, la contre-réaction résistive ayant une masse flottante $\mathrm{M}_{1}$, nous avons choisi une pile pour constituer la source de référence de haute stabilité $V_{0}$. Nous supprimons ainsi tout risque de ronfle à la fréquence du secteur. Nous évitons également d'introduire une impédance parasite par rapport à la masse qui perturberait le fonctionnement de la contre-réaction capacitive.

L'étude du système de contre-réaction capacitive ayant déjà été présentée [7], nous ne détaillerons ciaprès que la contre-réaction résistive.

3. Réalisation pratique de la contre-réaction résistive. - 3.1 TENSION DE RÉFÉRENCE. - Nous utilisons 


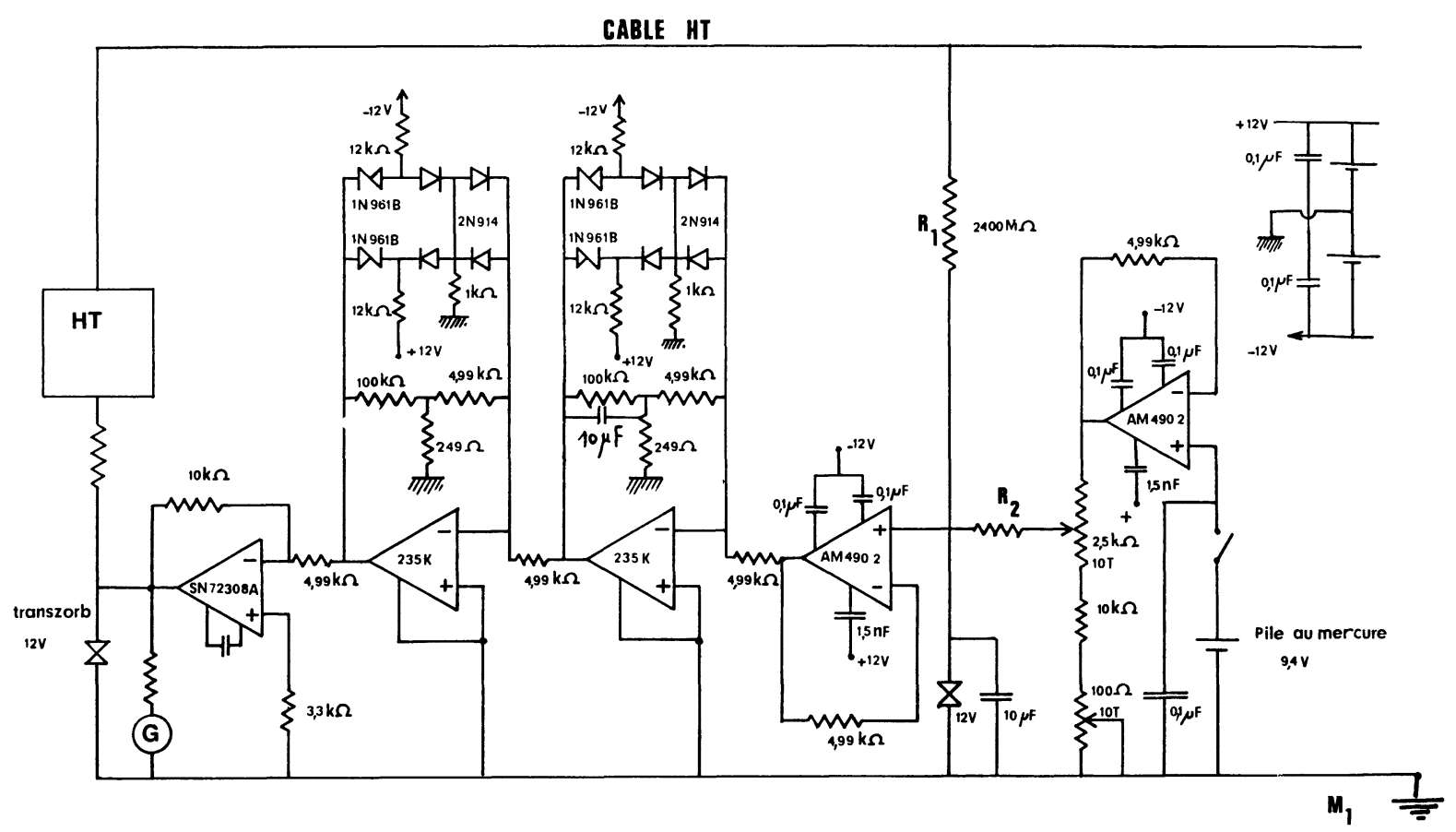

Fig. 2. - Schéma de montage de la contre-rćact.in résistive.

[Summarized circuit details of the resistif coupling seriesfeedback.]

une pile au mercure de très haute stabilité dont la tension est 9,4 V (Fig. 2). Elle est associée à un étage suiveur à très grande impédance d'entrée. L'amplificateur de l'étage suiveur est stabilisé par Chopper. Il présente un bruit de $6 \mu \mathrm{V}$ RMS de $10^{-2}$ à $10 \mathrm{~Hz}$. Sa dérive de tension d'offset est de $0,3 \mu \mathrm{V}$ par degré $\mathrm{C}$. Cet amplificateur opérationnel sert également d'adaptateur d'impédance pour séparer le diviseur résistif $\left(R_{1}, R_{2}\right)$ de l'étage d'amplification.

3.2 Diviseur RÉSISTIF. - Comme résistance $R_{1}$ nous utilisons la résistance de division de notre accélérateur d'électrons qui vaut environ $2400 \mathrm{M} \Omega$. Elle est constituée par une chaîne de 351 résistances Ohmic de 6,8 M $\Omega$ associées en série sur un mandrin isolant, l'ensemble étant surmoulé à l'araldite. Cette résistance est disposée dans l'enceinte de notre accélérateur pressurisée avec de l'hexafluorure de soufre à 1,6 bar.

La résistance $R_{2}$ comporte une série de résistances à couches métalliques de haute stabilité (SFERNICE RCMA K4) dont le coefficient de température est $15 \times 10^{-6}$ par degré C. L'ensemble $\left(R_{1}, R_{2}\right)$ constitue le diviseur résistif qui est placé à l'intérieur de l'enceinte pressurisée de notre accélérateur. L'inertie thermique de l'ensemble ne peut conduire dans la pratique qu'à des dérives thermiques très lentes qui ne sont pas gênantes en microscopie électronique.

L'expérience montrera d'ailleurs que la sensibilité du système aux variations de températures n'est pas une cause de détérioration de la stabilité.

3.3 Etage Amplificateur. - La formule (1) donne l'expression de la tension $V_{\mathrm{S} 1}$ en fonction de $V_{0}, V$, $A, R_{1}, R_{2}$ et de $d$ qui représente la somme des dérivés et des bruits de l'amplificateur $A$ ramenée à l'entrée de celui-ci, soit :

$$
\begin{aligned}
V_{\mathrm{S} 1}=\frac{V}{1+\frac{A R_{2}}{R_{1}+R_{2}}}-V_{0} \cdot \frac{R_{1}}{R_{2}} & \frac{1}{1+\frac{1}{A}\left(1+\frac{R_{1}}{R_{2}}\right)}- \\
& -\frac{d}{\frac{1}{A}+\frac{R_{2}}{R_{1}+R_{2}}} \cdot \text { (1) }
\end{aligned}
$$

Pour réduire au maximum la variation de $V_{\mathrm{S} 1}$ en fonction de $V$, il faut que $A$ soit très grand devant $\left(R_{1}+R_{2}\right) / R_{2}$ soit $A \gg R_{1} / R_{2}$ car $R_{2} \ll R_{1}$. Dans ce cas on peut écrire :

$$
\begin{aligned}
V_{\mathrm{S} 1} & \simeq V_{\mathrm{S}} \simeq-\left(V_{0} \frac{R_{1}}{R_{2}}+d \frac{R_{1}}{R_{2}}\right) \\
& \simeq-V_{0}\left(\frac{R_{1}}{R_{2}}\right) \quad \text { car } \quad d \ll V_{0} .
\end{aligned}
$$

Dans nos conditions expérimentales, nous avons $V_{0}=9,4 \mathrm{~V}$ et $V_{\mathrm{S}}=200 \mathrm{kV}$ ce qui implique que le gain de l'amplificateur devra être très grand devant $-V_{\mathrm{s}} / V_{0}$ soit $A \gg 20000$. Nous avons choisi un gain $A=400000$. Pour obtenir cette valeur de $A$, sans trop réduire la bande passante de l'amplificateur nous avons utilisé deux amplificateurs opérationnels du type $235 \mathrm{~K}$ Analog Devices, stabilisés par Chopper, dont le bruit ramené à l'entrée est de $3,5 \mu \mathrm{V}$ RMS de $10^{-1}$ à $10 \mathrm{~Hz}$ et dont la tension de dérive d'offset est de $0,25 \mu \mathrm{V}$ par degré C. Chaque amplificateur a un gain de 422 obtenu par un montage en $\mathrm{T}$ utilisant des résistances à couche métallique et dont la stabilité thermique est de $1.5 \times 10^{-6}$ par degré $C$. Pour éviter le blocage en 
saturation de ces amplificateurs nous utilisons un montage à diodes. Un troisième amplificateur opérationnel SN $72308 \mathrm{~A}$ assure la mise en opposition de phase du signal et élève le gain total à 400000 . Le bruit et la dérive ramenés à l'entrée de l'étage amplificateur sont respectivement de $10 \mu \mathrm{V}$ et de $0,9 \mu \mathrm{V}$ par degré $\mathrm{C}$.

4. Résultats expérimentaux. - Pour tester la boucle d'asservissement de la figure 1 nous avons introduit un signal d'erreur $\Delta V$ entre le câble haute tension et la masse flottante $M$ du générateur. Ce signal est fourni par un générateur basse fréquence associé à un photocoupleur. Sa valeur crête à crête est de 10 volts. Le signal de sortie $\Delta V_{\mathrm{S}}$ est mesuré sur un diviseur capacitif dont les caractéristiques ont été préalablement étalonnées en fonction de la fréquence. Les résultats obtenus sont présentés sur un diagramme (Fig. 3) qui donne la variation de l'atténuation - $20 \log \frac{\Delta V_{\mathrm{S}}}{\Delta V}$ en fonction de la fréquence du signal. Nous pouvons voir que dans une bande de fréquence allant de zéro à quelques Hertz l'atténuation est constante et vaut $-35 \mathrm{~dB}$. Dans cette première bande de fréquence c'est la contreréaction résistive qui fixe cette atténuation. Pour les fréquences supérieures, l'atténuation décroît lentement pour atteindre $-3 \mathrm{~dB}$ à quelques $\mathrm{kHz}$. Elle reste toutefois meilleure que $-20 \mathrm{~dB}$ entre 1 et $100 \mathrm{~Hz}$. Dans cette seconde bande de fréquence c'est la contre-réaction capacitive qui contribue à l'atténuation.

Nous avons ensuite mesuré l'amélioration pratique introduite par cette boucle d'asservissement lorsque le

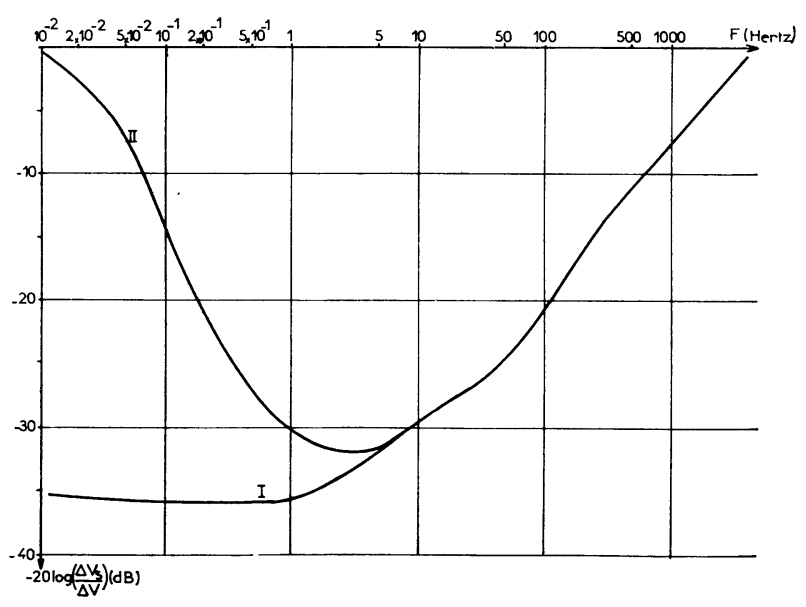

Fig. 3. - Diagramme d'atténuation de la boucle d'asservissement représentant les variations de l'atténuation $-20 \log \left(\Delta V_{\mathrm{S}} / \Delta V\right)$ en fonction de la fréquence dans deux cas différents. I. - La boucle d'asservissement comporte les deux dispositifs de contreréaction. II. - La boucle d'asservissement ne comporte que la contre-réaction à lecture capacitive. Pour les fréquences supérieures à $5 \mathrm{~Hz}$ les deux courbes sont confondues.

[Diagram showing the attenuation $\left(-20 \log \left(\Delta V_{\mathrm{S}} / \Delta V\right)\right.$ versus the frequency) given by the additional series-feedback circuit in two different cases. I. - The feedback circuit is made with both the resistif and capacitive coupling series-feedback circuits, both being connected in series. For frequencies above $5 \mathrm{~Hz}$, both curves are identical. II. - The feedback circuit is made with the capacitive coupling series-feedback circuit only.] générateur haute tension est en fonctionnement à $200 \mathrm{kV}$. Dans ce cas, le signal d'erreur $\Delta V$ est celui produit par le générateur. Il est constitué par une ondulation lente périodique à $0,2 \mathrm{~Hz}$ environ d'amplitude 3 volts crête à crête, à laquelle se superpose un bruit $\mathrm{BF} 50 \mathrm{~Hz}$ d'amplitude 5 volts crête à crête; il n'y a pratiquement pas de bruit haute fréquence car le générateur HT est du type électrostatique.

Nous précisons que dans ce test, nous avons mesuré le bruit $\mathrm{BF} 50 \mathrm{~Hz}$ à partir du diviseur capacitif précédemment utilisé, tandis que l'ondulation lente périodique a été captée entre un point de mesure situé au niveau du premier étage de l'amplificateur de la contreréaction résistive et la masse flottante $M_{1}$. Cette modification a été faite dans le but d'analyser avec une plus grande sensibilité les variations lentes de la tension délivrée par le générateur HT. Un interrupteur à deux positions ( 1 et 2 sur la figure 1 ) permet respectivement de supprimer ou d'introduire la contre-réaction résistive, tout en captant l'instabilité résiduelle sur le câble haute tension dans les deux cas.

Les résultats obtenus dans ces conditions sont représentés sur la figure 4. La boucle d'asservissement présentée sur la figure 1 , réduit le signal de sortie $\Delta V_{\mathrm{S}}$ à $150 \mathrm{~m} \mathrm{~V}$ pour l'ondulation $0,2 \mathrm{~Hz}$ et à $350 \mathrm{mV}$ pour le bruit $50 \mathrm{~Hz}$, les atténuations $-20 \log \frac{\Delta V_{\mathrm{s}}}{\Delta V}$ valant respectivement $-26 \mathrm{~dB}$ et $-23 \mathrm{~dB}$. La disparité des résultats entre les deux tests provient de l'influence de l'impédance de sortie du générateur HT. Ces résultats ne sont obtenus qu'au bout d'une heure environ de mise en équilibre du générateur HT; ce n'est que dans

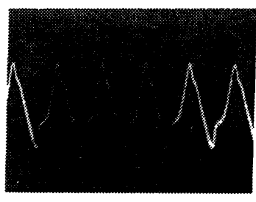

A 1

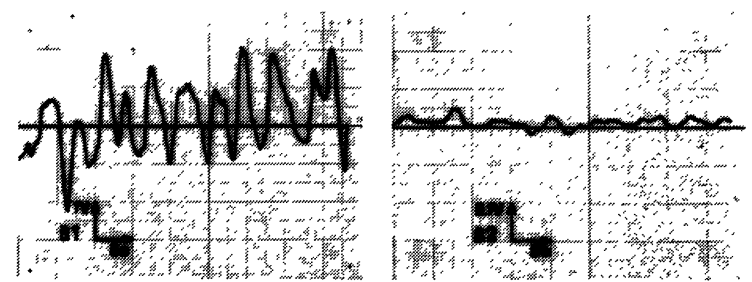

FIg. 4. - Signaux captés sur le câble HT illustrant l'amélioration introduite par la boucle d'asservissement schématisée figure 1, lorsque le générateur est en fonctionnement à $200 \mathrm{kV}$. A1. - Signal basse fréquence $(50 \mathrm{~Hz})$ sans la boucle. A2. Signal basse fréquence $(50 \mathrm{~Hz})$ avec la boucle. B1. - Signal très basse fréquence $(0,2 \mathrm{~Hz})$ sans la boucle. B2. - Signal très basse fréquence $(0,2 \mathrm{~Hz})$ avec la boucle.

[Ripple noises measured on the HV cable showing the improvement given by the additional feedback circuit (Fig. 1) when the HV generator is working at $200 \mathrm{kV}$. A1. - Low frequency ripple $(50 \mathrm{~Hz})$ without the feedback circuit. A2. - Low frequency ripple $(50 \mathrm{~Hz})$ with the feedback circuit. B1. - Very low frequency ripple $(0,2 \mathrm{~Hz})$ without the feedback circuit. B2. Very low frequency ripple $(0,2 \mathrm{~Hz})$ with the feedback circuit.] 
ces conditions que la boucle d'asservissement peut être mise en service, compte tenu de sa faible dynamique.

L'instabilité relative de l'ensemble est donc finalement ramenée de $2 \times 10^{-5}$ à $2 \times 10^{-6}$ et ceci pour toutes les fréquences allant de 0 à plus de $1000 \mathrm{~Hz}$.

5. Conclusion. - Nous avons montré qu'il était possible d'améliorer considérablement la stabilité relative en tension de notre générateur haute tension.
L'adaptation proposée se révèle être peu onéreuse dans la mesure où l'on dispose de la résistance $R_{1}$ du diviseur résistif ; ceci est généralement le cas lorsque ce problème concerne la microscopie électronique. Nous noterons enfin que nous avons fait nos tests à une tension moyenne de $200 \mathrm{kV}$, car le tube accélérateur de notre microscope électronique présente des instabilités pour des tensions supérieures. Le dispositif présenté peut toutefois être utilisé pour des tensions allant jusqu'à $400 \mathrm{kV}$.

\section{Bibliographie}

[1] Beorchia, A., Bonhomme, P., Optik 39 (1974) 437.

[2] ReInhold, G. et al., Z. Instr. 67 (1959) H. 10.

[3] Adler, H. et al., Proc. Evr. Reg. Conf. E. M. - Delft (1960).

[4] Dupouy, G. et al., C. R. Acad. Sci. Paris Ser. B 262 (1966) 341.
[5] Dupouy, G. et al., C. R. Acad. Sci. Paris Ser. B 269 (1969) 959.

[6] Genotel, D., Balossier, G., Severin, C., Girard, M., Homo, J. C., Laberrigue, A., C. R. Acad. Sc. Paris 272 Série B. (1971) 1461, 1464.

[7] GiraRd, M., Electronics letters Vol. 8 № 9 (1972). 